\title{
Findings About Events or Interventions Baseline Flag
}

National Cancer Institute

\section{Source}

National Cancer Institute. Findings About Events or Interventions Baseline Flag. NCI Thesaurus. Code C113359.

An indication or description that a finding about an event or intervention is a baseline value. 